\title{
TECHNOLOGIES FOR OBTAINING RADIO-PURE MATERIALS; METHODS OF LOW RADIOACTIVITY DETECTION*
}

\author{
KRZYSZTOF PELCZAR \\ The Marian Smoluchowki Institute of Physics, Jagiellonian University \\ Reymonta 4, 30-059 Kraków, Poland
}

(Received August 6, 2013)

This paper discusses selected problems arising from the presence of radioactive background sources in experiments searching for extremely rare processes. Physical properties of ${ }^{42} \mathrm{~K}$ ions, present in argon as a progeny of ${ }^{42} \mathrm{Ar}$, were investigated. A model of ${ }^{42} \mathrm{~K}$ ions behavior in liquiefied argon is presented. Also, construction and operation of an electrostatic ${ }^{222} \mathrm{Rn}$ monitor of gaseous nitrogen is outlined.

DOI:10.5506/APhysPolBSupp.6.1073

PACS numbers: 23.60.+e, 29.30.Ep, 29.30.Kv

\section{Introduction}

Liquefied noble gases are extensively used in ultra-low background experiments searching for rare processes. The cryogenic liquids serve as a radiopure material for passive shielding and as a cooling medium (liquefied argon in the GERDA experiment [1]) or both as a target and detector (two phase argon TPC in the DarkSide experiment [2]). The ultra-low background experiments are aiming at maximization of their sensitivity to registering rare events by mitigating any possible background sources. Careful material selection and on-line monitoring for background sources is, therefore, crucial. E.g. the detection limit for half life-time of ${ }^{76} \mathrm{Ge}$ regarding neutrinoless double beta decay $(0 \nu 2 \beta)$ is inversely proportional to the square root of the background index $B_{E}$

$$
T_{1 / 2}^{0 \nu}>A \epsilon \frac{\ln 2}{m_{\mathrm{mol}}} \sqrt{\frac{m T}{B_{E} \delta_{E}}} N_{\mathrm{A}},
$$

\footnotetext{
* Presented at the Symposium on Applied Nuclear Physics and Innovative Technolo-
} gies, Kraków, Poland, June 3-6, 2013. 
where $A$ is the ${ }^{76} \mathrm{Ge}$ isotope abundance, $\epsilon$ is the $0 \nu 2 \beta$ decay registration efficiency, $m_{\mathrm{mol}}$ is the molar mass of Ge, $m$ is the detector mass, $T$ is the duration of measurement, $B_{E}$ is the background index in region of interest (at expected $0 \nu 2 \beta$ decay energy), $\delta_{E}$ is the energy resolution, and $N_{\mathrm{A}}$ is the Avogadro number. Therefore, the most effort is put forth in lowering the experimental background.

\section{2. ${ }^{42} \mathrm{~K}$ in liquid argon}

${ }^{42} \mathrm{~K}$ is produced in beta decays of ${ }^{42} \mathrm{Ar}$, naturally present in argon $\left({ }^{42} \mathrm{Ar} /{ }^{\text {nat }} \mathrm{Ar}<3 \times 10^{-21} \mathrm{~g} / \mathrm{g}\right)$. High energy released in beta decay of ${ }^{42} \mathrm{~K}$ $(Q=3525 \mathrm{keV})$ exceeds the ${ }^{76} \mathrm{Ge} 0 \nu 2 \beta$ decay energy $(Q=2039 \mathrm{keV})$, therefore, ${ }^{42} \mathrm{~K}$ is a potential source of background for e.g. the GERDA experiment.

Potassium may form positive ions as a result of ${ }^{42} \mathrm{Ar}$ beta decays occurring in the cryogenic liquid. Electric potential present in the active volume of a detector (biased bare germanium diodes in the GERDA experiment or TPC in the DarkSide experiment) is responsible for transport of the ions. In consequence, initial concentration of the impurities homogeneously distributed in the volume of the detector may be altered. Figure 1 presents the described processes.

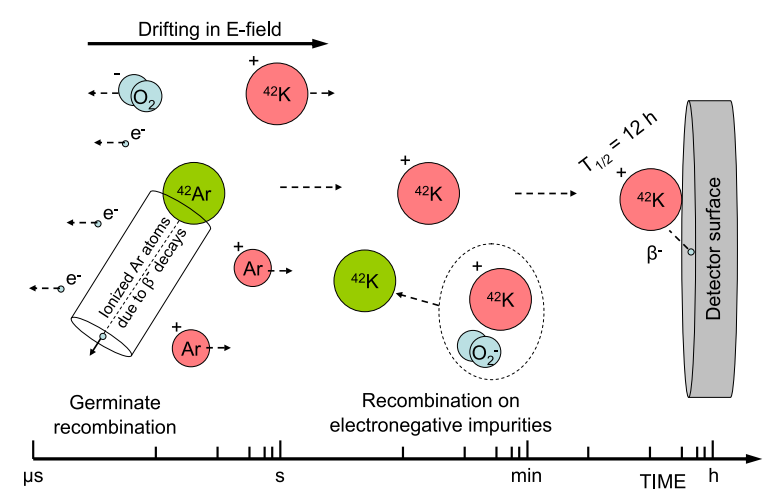

Fig. 1. Model of ${ }^{42} \mathrm{~K}$ drifting in liquefied argon containing traces of ${ }^{42} \mathrm{Ar}$ in presence of an external electric field. Initially, ${ }^{42} \mathrm{~K}$ is produced as a cation in a beta decay of ${ }^{42} \mathrm{Ar}$. Within its life-time, the cation may be drifted towards the active volume of a detector. The drift distance is limited by neutralization of the cation by electronegative impurities.

To fully understand the radioactive contribution of ${ }^{42} \mathrm{~K}$ beta decays to the background, one has to study the germinate recombination processes and transport properties of the cations. The germinate recombination is responsible for immediate neutralization of the cations following the beta decay. This process determines, depending on the external electric field strength, 
the amount of cations retaining their charge. Spatial range of the fraction of ions surviving the germinate neutralization varies with mobility and ionic half life-time of the ions (neutralization of the ions by electronegative impurities like oxygen). The properties of ${ }^{42} \mathrm{~K}$ were extensively studied in dedicated experiments (Liquid Argon Germanium facility in the framework of the GERDA experiment - LArGe).

\section{3. ${ }^{222} \mathrm{Rn}$ and its progenies}

The DarkSide experiment is searching for interactions of Cold Dark Matter particles (Weakly Interacting Massive Particles - WIMPs) with atoms of liquefied argon. The signal is detected by a two-phase Time Projection Chamber. Alpha decays of ${ }^{222} \mathrm{Rn}$ progenies may mimic the signal from WIMPs, serving as a potential source of the experimental background.

${ }^{222} \mathrm{Rn}$ belongs to the decay chain of ${ }^{226} \mathrm{Ra}$, present in all materials used for construction of the experiments. Radon is diffusing and emanating from surfaces of materials, being an inert noble gas. ${ }^{222} \mathrm{Rn}$ may also be ejected from thin surface layers of a material as a recoil atom of ${ }^{226} \mathrm{Ra}$ alpha decay. ${ }^{222} \mathrm{Rn}$ then enters active volumes of the detectors by dissolving in other gases easily. Figure 2 shows the ${ }^{222} \mathrm{Rn}$ decay chain.

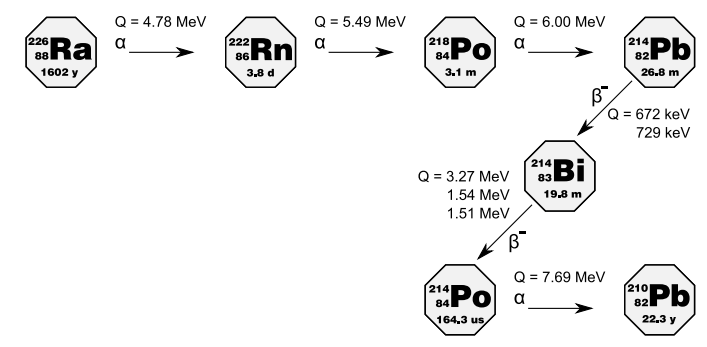

Fig. 2. ${ }^{222} \mathrm{Rn}$ decay chain.

Figure 3 depicts a basic concept of an on-line ${ }^{222} \mathrm{Rn}$ activity detector for gases. The radioactive cations produced in decays of ${ }^{222} \mathrm{Rn}$ progenies' present inside the investigated gas are being drifted in the external electric field (positively biased housing of the detector chamber up to $+20 \mathrm{kV}$ ) towards the alpha detector (grounded). The cations are then embedded on the detectors surface. The Si-PIN detector registers alpha decays of the collected cations $\left({ }^{218} \mathrm{Po}\right.$ and $\left.{ }^{214} \mathrm{Po}\right)$.

The electrostatic ${ }^{222} \mathrm{Rn}$ monitor (400l volume) was recently constructed and deployed to operation for the DarkSide experiment. ${ }^{222} \mathrm{Rn}$ content is instantly controlled in various gases used in the experiment: air exhausted from clean-rooms, nitrogen and argon. ${ }^{222} \mathrm{Rn}$ concentration detection limit achieved by the device is better than $10 \mathrm{mBq} / \mathrm{m}^{3}$. 


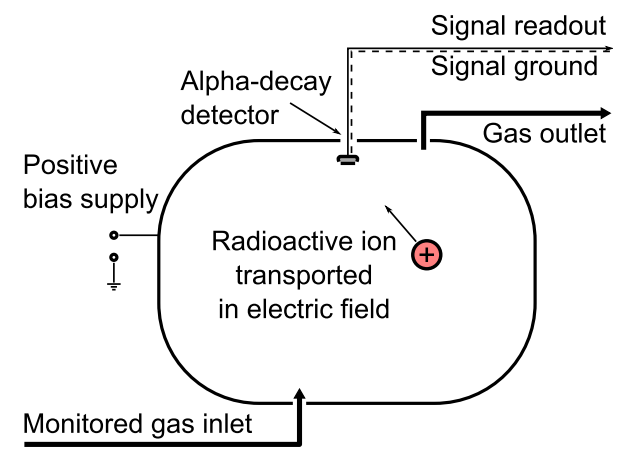

Fig. 3. Electrostatic detector of alpha-decaying ${ }^{222} \mathrm{Rn}$ daughters in gases. ${ }^{222} \mathrm{Rn}$ present in monitored gas is transported to the detector chamber. In series of alpha and beta decays, ${ }^{222} \mathrm{Rn}$ progenies are produced as positively charged ions. The positive high voltage bias applied to the detector chamber repels the ions towards the grounded alpha decay detector. The Si-PIN detector registers alpha decays of ${ }^{218} \mathrm{Po}$ and ${ }^{214} \mathrm{Po}$.

\section{Conclusions}

Ultra-low background experiments demand supreme purities of the construction materials. Also, during their operation on-line monitoring of the background index is essential. Technologies for obtaining highly radiopure materials rely on careful material selection and understanding of the physical properties of the radio-impurities. Dedicated experiments focused only on the impurities need to be designed to study their nature.

We acknowledge support by the Foundation for Polish Science - MPD program, co-financed by the European Union within the European Regional Development Fund. This work was also supported by a grant from the National Centre for Research and Development in the frame of the ERANET ASPERA II Programme. We also acknowledge financial support by the Jagiellonian University (Institute of Physics, DSC 2012).

\section{REFERENCES}

[1] K.H. Ackermann et al. [GERDA Collaboration], Eur. Phys. J. C73, 2330 (2013).

[2] The DarkSide Collaboration, DarkSide-50 Proposal (2008),

http://darkside.lngs.infn.it/ 